\title{
El programa multicomponente de apoyo para el cese del tabaquismo (MCSCSP) es efectivo en pacientes con trastorno mental grave sin diferencias de género
}

\section{The Multi-Component Smoking Cessation Support Programme (McSCSP) is effective in patients with severe mental disorder without gender differences}

\author{
María José Jaén-Moreno*,**, María Paz García-Portilla****************************, Fernando \\ SarrameA***************, Teresa Bobes Bascarán***,************************, GonZalo \\ Galván***********, Eva María Díaz-MesA************, Susana Al-Halabi*******,*********, \\ Edorta Elizagarate*************, Pilar Alejandra Sáiz Martínez***,****,*****,******,*******, \\ Julio Bobes $* * *, * * * *, * * * * *, * * * * * *, * * * * * * *$, Leticia García-ÁldVAREZ***,****,*****,******************. \\ * Instituto Maimónides de Investigación Biomédica de Córdoba (IMIBIC), Córdoba, España. \\ ** Departamento de Ciencias Sociosanitarias, Radiología y Medicina Física, Universidad de Córdoba, Córdoba, España. \\ *** Instituto de Investigación Sanitaria del Principado de Asturias (ISPA), Oviedo, Asturias, España. \\ ***** Área de Psiquiatría, Universidad de Oviedo, Oviedo, Asturias, España. \\ ***** Centro de Investigación Biomédica en Red de Salud Mental (CIBERSAM), Oviedo, Asturias, España. \\ ****** Servicio de Salud del Principado de Asturias (SESPA), Oviedo, Asturias, España. \\ ******* Instituto de Neurociencias del Principado de Asturias (INEUROPA), Asturias, España. \\ ******** Unidad de Gestión Clínica de Salud Mental, Hospital Universitario Reina Sofía, Córdoba, España. \\ ********* Área de Personalidad, Evaluación y Tratamiento Psicológico, Universidad de Oviedo, Oviedo, Asturias, España. \\ *********** Centro de Investigación Biomédica en Red de Salud Mental (CIBERSAM), Valencia, España. \\ ************ Universidad Cooperativa de Colombia, Colombia. \\ ************* Universidad Autónoma de Madrid, Madrid, España. \\ ************** Unidad de Psicosis Refractarias, Hospital Psiquiátrico de Álava, Vitoria, España.
}

\section{Resumen}

La elevada prevalencia del tabaquismo en personas con trastorno mental grave (TMG) contribuye a su morbilidad médica y reduce su esperanza de vida. A pesar de la existencia de diferencias de género en el cese del tabaquismo, pocos estudios han evaluado esas diferencias en personas con TMG. Este es un ensayo multicéntrico de seguimiento prospectivo, no aleatorizado, abierto de 9 meses para examinar las diferencias de género en la eficacia, seguridad y tolerabilidad de un programa multicomponente de apoyo para el cese del tabaquismo (McSCSP). Los resultados mostraron que no hubo diferencias de género significativas en la eficacia a corto (hombres $44,9 \%$ vs mujeres $57,7 \%$, chi cuadrado $=1,112, p=$ ,292) ni a largo plazo (semana 24 : hombres $40,8 \%$, mujeres $42,0.3 \%$, chi cuadrado $=0.016, p=, 901$; semana 36 : hombres $36,7 \%$, mujeres $38,5 \%$,

\begin{abstract}
High prevalence of smoking in people with severe mental disorders (SMD) contributes to their medical morbidity and reduced life expectancy. Despite the evidence of gender differences in smoking cessation, few studies have tested those differences among people with SMD. This is a non-randomized, open-label, prospective, 9-month follow-up multicentre trial to examine gender differences in the efficacy, safety and tolerability of a Multi-Component Smoking Cessation Support Programme (McSCSP). The results showed that there were no significant differences in short- (males $44.9 \%$ vs females $57.7 \%$, chi-square $=1.112, p=0.292$ ) or long-term efficacy (week 24 : males $40.8 \%$, females $42.3 \%$, chi-square $=0.016, p=0.901$; week 36 : males $36.7 \%$, females $38.5 \%$, chi-square $=0.022$, $p=0.883$ ) between
\end{abstract}


chi cuadrado $=0,022, \mathrm{p}=, 883)$, incluso controlando por diagnóstico o tratamiento. Con respecto a la seguridad y la tolerabilidad, hubo un aumento significativo en el perímetro abdominal en los hombres [de $105,98$ (DT 13,28) a 108,52 (DT 14,01), $t=-3,436, p=, 002)$ ], pero no en las mujeres. Sin embargo, no hubo diferencias de género significativas en los eventos adversos (estreñimiento, sueños anormales/vívidos, náuseas/vómitos o erupción cutánea/enrojecimiento alrededor de la zona del parche). En conclusión, hemos demostrado que es efectivo y seguro ayudar a los hombres y mujeres con TMG estabilizados a dejar de fumar. Sin embargo, podría haber una tendencia en las mujeres a responder mejor al tratamiento con vareniclina a corto plazo. Se requiere investigación futura con muestras más amplias para determinar con más claridad la existencia de diferencias, además de la fiabilidad y robustez.

Palabas clave. Diferencias de género; cese del tabaquismo; esquizofrenia; trastorno bipolar; vareniclina; parches transdérmicos de nicotina.

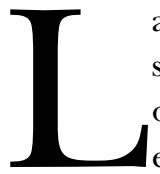

a prevalencia estimada del tabaquismo en personas con trastornos mentales graves (TMG) y otros trastornos es entre $50-80 \%$ y $54-68 \%$ para esquizofrenia y trastorno bipolar, respectivamente (De Hert et al., 2011; Jiménez-Treviño et al., 2019; Rodriguez Muñoz, Carmona Torres, Hidalgo Lopezosa, Cobo Cuenca y Rodríguez Borrego, 2019). En algunos países, las tasas de tabaquismo son similares entre hombres y mujeres con trastornos psicóticos; en otros, como Asia, las mujeres con esquizofrenia tienen menos probabilidades de ser fumadoras activas que los hombres (Hahn, Rigby y Galletly, 2014; Kim et al., 2013). El tratamiento multicomponente en entornos clínicos ha demostrado la importancia del nivel motivacional durante la fase preparatoria (Garcia-Portilla et al., 2016; Sarramea Crespo et al., 2019a; Sarramea et al., 2019b).

$\mathrm{Al}$ examinar las diferencias de género en los resultados sobre cesación tabáquica en población general, se han encontrado algunas diferencias entre hombres y mujeres. Las mujeres son menos propensas a usar la terapia de reemplazo de nicotina (TRN) (Perkins, 2001), tienden a tener más dificultades para dejar de fumar (Perkins, 2001; Reid, Pipe, Riley y Sorensen, 2009; Walker et al., 2016) y muestran resultados del tratamiento para dejar de fumar más pobres en ensayos de tratamiento con muestras amplias de población (Bjornson et al., 1995; COMMIT, 1995) tanto con Bupropion (Scharf y Shiftman, 2004) como con TRN (Davis et al., 1994; Perkins y Scott, 2008; Wetter et al., 1999). Sin embargo, el único estudio llevado a cabo con pacientes con psicosis no encontró diferencias de género en esas variables (Filia et al., 2014). Por tanto, se ne- gender, neither controlled by diagnosis or treatment. Regarding safety and tolerability, there was significant increase in abdominal perimeter in males [from 105.98 (SD 13.28) to 108.52 (SD 14.01), $t=-3.436$, $p=0.002)]$, but not in females. However, there were no significant gender differences in adverse events (constipation, abnormal/vivid dreams, nausea/vomiting or skin rash/redness around patch site). In conclusion, we have demonstrated that is effective and safe to help either male or female patients with stabilized SMD to quit smoking. However, it might be a tendency in females to respond better to varenicline treatment in the short-term. Future research with larger samples is required to more clearly determine whether or not the there are differences, in addition to their reliability and robustness. Keywords: Gender differences; smoking cessation; shizophrenia; bipolar disorder; varenicline; transdermal nicotine patches.

cesita más investigación sobre este tema para determinar qué programas podrían ser más efectivos en función del género.

El objetivo de este estudio fue examinar las diferencias de género en la eficacia, seguridad y tolerabilidad de un programa multicomponente de apoyo para el cese del tabaquismo (McSCSP) (Garcia-Portilla et al., 2014, 2016) diseñado específicamente para el tratamiento de pacientes con TMG bajo condiciones clínicas reales.

\section{Método}

\section{Diseño del estudio}

Este es un estudio multicéntrico, no aleatorio, abierto, prospectivo, de seguimiento de 9 meses, realizado en 3 ciudades de España (Oviedo, Jaén y Vitoria) entre marzo de 2011 y junio de 2013 (ver Garcia-Portilla et al., 2014, 2016). El Comité de Ética en Investigación Clínica del Hospital de Oviedo aprobó el protocolo de estudio (Ref. 64/2010).

El McSCSP consistió de 2 fases: la fase 1, terapia motivacional individual semanal durante 4 a 12 semanas antes de la fase de tratamiento activo, y la fase 2 , fase de tratamiento activo de 12 semanas. Durante la fase de tratamiento activo, los pacientes recibieron medicación y una terapia grupal intensiva manualizada de 12 semanas sobre cuestiones relevantes para ellos. La elección de tratamiento farmacológico de cada paciente se decidió conjuntamente entre médico y paciente (ver Garcia-Portilla et al., 2014 para más detalles). 


\section{Sujetos}

Los sujetos eran pacientes con diagnóstico de TMG clínicamente estables que estaban en tratamiento ambulatorio y asistían a sus consultas programadas. Durante esas citas, sus psiquiatras les ofrecían la posibilidad de participar en un estudio sobre el cese del tabaquismo.

Criterios de inclusión: diagnóstico de esquizofrenia según el DSM-IV, trastorno esquizoafectivo o bipolar; fumador actual de $\geq 15$ cigarrillos/día; puntuación $\geq 4$ en el test de Fagerström de dependencia de nicotina; carboximetría CO > 9 ppm; 18-65 años; sin ideación suicida; consentimiento informado por escrito.

Criterios de exclusión: puntuación total PANSS $>70$ (esquizofrenia) o puntuación HDRS $>14$ o YMRS $>6$ (trastorno bipolar); comportamiento/pensamientos suicidas graves en los últimos 6 meses; enfermedad somática inestable grave; daño cerebral orgánico; insuficiencia renal (creatinina $\geq 1,5 \mathrm{mg} / \mathrm{dL}$ ); y función hepática alterada (dos veces el límite superior normal).

\section{Evaluaciones}

Todos los sujetos fueron evaluados al inicio del estudio, durante la fase de tratamiento activo de 12 semanas, y en las semanas de seguimiento 12 y 24 de postratamiento. Se clasificaron en tres categorías de acuerdo al número de cigarrillos fumados por día $(\mathrm{CPD})$ : ligero $(\mathrm{CPD} \leq 10)$, moderado (entre 11 y 20), y grave ( $>20$ ). Para más detalles relacionados con la evaluación, ver Garcia- Portilla et al., (2014, 2016).

\section{Medidas de resultado y análisis estadísticos}

Las medidas de resultados principales de la semana 12 fueron las diferencias de género en el cese del tabaquismo (abstinencia autoinformada en los 7 días previos, confirmada por niveles de $\mathrm{CO}$ en carboximetría $\leq 9 \mathrm{ppm}$ ) y en la proporción de sujetos con una reducción mínima del
$50 \%$ en la cantidad de cigarrillos por día (CPD) durante los 7 días previos. Las medidas de resultado secundarias fueron las diferencias de género en la seguridad, incluidos los cambios en los síntomas de la enfermedad primaria, los intentos de suicidio y la tolerabilidad.

Se utilizó el SPSS 17.0 con un nivel de significanción de, 05 . Todos los análisis se realizaron de acuerdo con un enfoque de intención de tratar. Se empleó el método de la última observación realizada (LOCF) para tratar con datos faltantes. Las pruebas chi-cuadrado, $t$ de Student y t de muestras emparejadas se usaron para determinar las diferencias estadísticamente significativas entre géneros y para evaluar los cambios a lo largo del tiempo entre el inicio del estudio y la semana 12.

Se realizó un análisis de varianza mixto inter-intra sujetos para evaluar el impacto del género en el hábito tabáquico y en las variables clínicas en cuatro momentos (inicio del estudio, fase de tratamiento activo de 12 semanas, y en las semanas de seguimiento 12 y 24 del postratamiento).

\section{Resultados}

De los 82 pacientes incluidos en el estudio, se analizaron 75 [ (36 parches transdérmicos de nicotina (PTN) y 39 vareniclina; $72 \%$ esquizofrenia/trastorno esquizoafectivo y $28 \%$ trastorno bipolar; $65,3 \%$ hombres, $34,7 \%$ mujeres (chi cuadrado $=4,041, p=, 044)]$. Las tasas de retención del estudio fueron $61,3 \%$ (67,3\% hombres, $53,8 \%$ mujeres, chi cuadrado $=1,323, p=, 250$ ) en la semana $12,48 \%$ (49,0\% hombres, $46,2 \%$ mujeres, chi cuadrado $=0,054, p$ $=, 816)$ en la semana 24 y $46,6 \%$ (46,9\% hombres, $46,2 \%$ mujeres, chi cuadrado $=0,004, p=, 948)$ en la semana 36 . No hubo diferencias estadísticamente significativas en las tasas de retención entre géneros ni entre tratamientos. La Tabla 1 muestra el análisis descriptivo al inicio del estudio.

Tabla 1. Características demográficas, clínicas y de tabaquismo de los pacientes al inicio del estudio para la muestra total y para hombres y mujeres por separado.

\begin{tabular}{|c|c|c|c|c|}
\hline & $\begin{array}{l}\text { Muestra total } \\
\qquad N=75\end{array}$ & $\begin{array}{c}\text { Hombres } \\
n=49\end{array}$ & $\begin{array}{c}\text { Mujeres } \\
n=26\end{array}$ & Prueba estadística, $p$ \\
\hline Edad media (DT) & $45,3(9,0)$ & $44,1(9,7)$ & $47,8(7,2)$ & $2,957^{\mathrm{d}},, 090$ \\
\hline Estado civil [n (\%)] & & & & $17,635^{\mathrm{e}},<, 0001$ \\
\hline Nunca casado/o & $47(62,7)$ & $39(79,6)$ & $8(30,8)$ & \\
\hline Casado/a o viviendo en pareja & $16(21,3)$ & $5(10,2)$ & $11(42,3)$ & \\
\hline Viudo/a, separado/a o divorciado/a & $12(16,0)$ & $5(10,2)$ & $7(26,9)$ & \\
\hline Nivel de estudios [n (\%)] & & & & $2,500^{\mathrm{e}},, 287$ \\
\hline Primarios & $32(42,7)$ & $18(36,7)$ & $14(53,8)$ & \\
\hline Secundarios & $32(42,7)$ & $24(49,0)$ & $8(30,8)$ & \\
\hline Universitarios & $11(14,7)$ & $7(14,3)$ & $4(15,4)$ & \\
\hline Situación laboral [n (\%)] & & & & $6,043^{\mathrm{e}},, 110$ \\
\hline Empleado/a (jornada completa/parcial) & $7(9,3)$ & $5(10,2)$ & $2(7,7)$ & \\
\hline Incapacitado/a (temporal/permanente) & $33(44,0)$ & $26(53,1)$ & $7(26,9)$ & \\
\hline Prestación por enfermedad & $19(25,3)$ & $9(18,4)$ & $10(38,5)$ & \\
\hline Otro $^{\mathrm{a}}$ & $16(21,3)$ & $9(18,4)$ & $7(26,9)$ & \\
\hline
\end{tabular}


María José Jaén, María Paz García-Portilla, Fernando Sarramea, Teresa Bobes, Gonzalo Galván, Eva María Díaz-Mesa, Susana Al-Halabi, Edorta Elizagarate, Pilar Alejanda Sáiz, Julio Bobes, Leticia García-Álvarez

Tabla 1 (cont.). Características demográficas, clínicas y de tabaquismo de los pacientes al inicio del estudio para la muestra total y para hombres y mujeres por separado.

\begin{tabular}{|c|c|c|c|c|}
\hline & $\begin{array}{l}\text { Muestra total } \\
\qquad N=75\end{array}$ & $\begin{array}{c}\text { Hombres } \\
n=49\end{array}$ & $\begin{array}{c}\text { Mujeres } \\
n=26\end{array}$ & Prueba estadística, $p$ \\
\hline Diagnóstico [n (\%)] & & & & $4,041^{\mathrm{e}},, 044$ \\
\hline Esquizofrenia & $54(72,0)$ & $39(79,6)$ & $15(57,7)$ & \\
\hline Trastorno bipolar & $21(28,0)$ & $10(20,4)$ & $11(42,3)$ & \\
\hline Duración de enfermedad, meses [Media $(d t)]$ & $209,2(125,4)$ & $201,8(127,1)$ & $223,2(123,5)$ & $, 004^{\mathrm{d}},, 950$ \\
\hline Primer episodio, sí [n (\%)] & $10(13,5)$ & $5(10,4)$ & $5(19,2)$ & $1,121^{\mathrm{e}},, 290$ \\
\hline TUS comórbido [n (\%)] & $10(13,3)$ & $7(14,3)$ & $3(11,5)$ & $, 111^{\mathrm{e}},, 739$ \\
\hline \multicolumn{5}{|l|}{ Intentos de suicidio } \\
\hline Sí [n (\%)] & $29(38,7)$ & $15(30,6)$ & $14(53,8)$ & $3,867^{\mathrm{e}}, 049$ \\
\hline Número medio $(d t)$ & $2,8(1,8)$ & $2,6(1,2)$ & $2,9(2,3)$ & $3,992^{\mathrm{d}},, 056$ \\
\hline CGI-S [Media $(d t)]$ & $3,5(1,0)$ & $3,6(1,0)$ & $3,5(1,0)$ & $.022^{\mathrm{d}}, 884$ \\
\hline \multicolumn{5}{|l|}{$\operatorname{PANSS}^{\mathrm{b}}[$ Media $(d t)]$} \\
\hline Positivo & $11,4(3,8)$ & $11,5(3,8)$ & $11,1(4,0)$ & $, 020^{\mathrm{d}},, 887$ \\
\hline Negativo & $14,9(5,6)$ & $15,1(5,0)$ & $14,4(7,1)$ & $920^{\mathrm{d}},, 342$ \\
\hline Psicopatología general & $27,2(8,2)$ & $26,3(5,9)$ & $29,5(12,4)$ & $2,326^{\mathrm{d}},, 133$ \\
\hline Total & $52,2(11,4)$ & $52,9(11,1)$ & $50,3(12,4)$ & $, 146^{\mathrm{d}},, 704$ \\
\hline $\operatorname{HDRS}^{\mathrm{C}}[$ Media $(d t)]$ & $5,1(4,0)$ & $5,6(4,3)$ & $4,6(3,8)$ & $, 322^{\mathrm{d}},, 577$ \\
\hline YMRS' [Media $(d t)]$ & $2,9(2,5)$ & $1,9(2,7)$ & $3,7(2,1)$ & $1,095^{\mathrm{d}},, 308$ \\
\hline CPD autoinformado [Media $(d t)]$ & $30,1(11,8)$ & $31,4(12,7)$ & $27,7(9,8)$ & $2,111^{\mathrm{d}}, 0,150$ \\
\hline Estado de tabaquismo & & & & $0,687^{\mathrm{e}}, 0,407$ \\
\hline Moderado (CPD 11-20 autoinformado) & $27(36,0)$ & $16(32,7)$ & $11(42,3)$ & \\
\hline Crómico (CPD >20 autoinformado) & $48(64,0)$ & $33(67,3)$ & $15(57,7)$ & \\
\hline Niveles de CO en aliento & $27,3(18,3)$ & $30,0(20,6)$ & $22,1(11,4)$ & $4,636^{\mathrm{d}}, 0,035$ \\
\hline Puntuación FTND & $6,3(2,6)$ & $6,1(2,7)$ & $6,7(2,4)$ & $0,484^{d}, 0,489$ \\
\hline Puntuación G-NSBQ & $17,8(6,9)$ & $17,6(7,3)$ & $18,2(6,0)$ & $0,405^{\mathrm{d}}, 0,526$ \\
\hline Tratamiento & & & & $0,517^{\mathrm{e}}, 0,472$ \\
\hline PTN & $36(48,0)$ & $25(51,0)$ & $11(42,3)$ & \\
\hline Vareniclina & $39(52,0)$ & $24(49,0)$ & $15(57,7)$ & \\
\hline
\end{tabular}

Nota. CGI-S: escala de impresión clínica global - severidad; CO: monóxido de carbono; CPD: cigarrillos por día; FTND: test de Fagerström de dependencia de nicotina; GN-SBQ: Test de Glover-Nilsson; HDRS: escala de depresión de Hamilton; PANSS: Escala de Síntomas Positivos y Negativos; dt: desviación típica; TUS: trastorno por uso de sustancias; PTN: parches transdérmicos de nicotina; YMRS: escala de manía de Young.

a Otros incluye: desempleo, ama de casa, estudiante y jubilado/a.

${ }^{\mathrm{b}}$ Los datos para PANSS son de pacientes con esquizofrenia $(n=54)$.

c Los datos para HDRS y YMRS son de pacientes con trastorno bipolar $(n=21)$.

d Prueba $t$ de Student.

e Test de chi cuadrado.

\section{Eficacia}

La Figura 1 muestra la eficacia tanto a corto como largo plazo para hombres y mujeres. No hubo diferencias significativas en la eficacia a corto y a largo plazo en función del género, diagnóstico o tratamiento. Además, el efecto de interacción entre el tiempo y el género no fueron significativas para ninguna de las variables (Tabla 2).

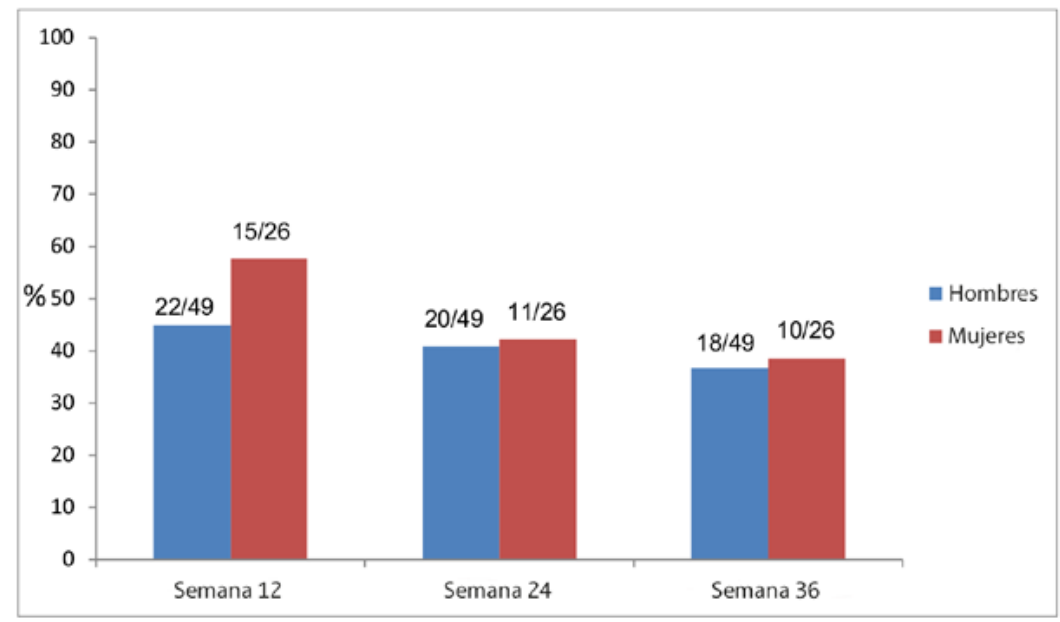

Figura 1. Eficacia a corto y largo plazo ${ }^{\mathrm{a}}$ para hombres y mujeres.

Nota. No hubo diferencias estadísticamente significativas en las tasas de eficacia entre hombres y mujeres en cualquier período temporal. ${ }^{a}$ Informe autoinformado por el paciente de la abstinencia durante los 7 días anteriores confirmado por niveles de $\mathrm{CO} \leq 9 \mathrm{ppm}$. 
El programa multicomponente de apoyo para el cese del tabaquismo (McSCSP) es efectivo en pacientes con trastorno mental grave sin diferencias de género

Tabla 2. Características del tabaquismo para hombres y mujeres en los cuatro períodos temporales.

\begin{tabular}{|c|c|c|c|c|c|c|c|c|}
\hline & \multicolumn{4}{|c|}{ Hombres } & \multicolumn{4}{|c|}{ Mujeres } \\
\hline & $\begin{array}{l}\text { Inicio del } \\
\text { estudio }\end{array}$ & $\mathrm{S}_{12}$ & $\mathrm{~S} 24$ & $\mathrm{~S}_{36}$ & $\begin{array}{l}\text { Inicio del } \\
\text { estudio }\end{array}$ & S12 & $\mathrm{S} 24$ & $S_{36}$ \\
\hline \multicolumn{9}{|l|}{ [media $(d t)]$} \\
\hline CPD & $31,4(12,7)$ & $6,8(8,7)$ & $9,6(10,5)$ & $9,1(9,0)$ & $27,7(9,8)$ & $7,9(12,3)$ & $10,4(12,2)$ & $10,3(12,4)$ \\
\hline Nivel de CO en aliento & $30,0(20,6)$ & $12,4(14,6)$ & $13,6(14,7)$ & $14,4(15,6)$ & $22,1(11,4)$ & $9,2(12,6)$ & $11,9(13,2)$ & $12,2(12,0)$ \\
\hline Puntuaciones en FTND & $6,1(2,7)$ & $2,6(3,1)$ & $2,8(3,1)$ & $3,2(3,0)$ & $6,7(2,4)$ & $2,2(3,1)$ & $2,9(3,5)$ & $3,2(3,4)$ \\
\hline Puntuaciones en GN-SBQ & $17,6(7,3)$ & $9,0(8,9)$ & $9,2(9,1)$ & $9,7(9,8)$ & $18,2(6,0)$ & $7,5(7,7)$ & $9,4(8,8)$ & $10,2(7,8)$ \\
\hline \multicolumn{9}{|l|}{ Fumador $^{\mathrm{a}}$ [n (\%)] } \\
\hline Abstinente & & $24(49,0)$ & $21(42,9)$ & $18(38,8)$ & & $16(61,5)$ & $12(46,2)$ & $12(46,2)$ \\
\hline Leve & & $10(20,4)$ & $6(12,2)$ & $10(20,4)$ & & $2(7,7)$ & $3(11,5)$ & $4(15,4)$ \\
\hline Moderado & $16(32,7)$ & $12(24,5)$ & $16(32,7)$ & $17(34,7)$ & $11(42,3)$ & $4(15,4)$ & $7(26,9)$ & $5(19,2)$ \\
\hline Grave & $33(67,3)$ & $3(6,1)$ & $6(12,2)$ & $3(6,1)$ & $15(57,7)$ & $4(15,4)$ & $4(15,4)$ & $5(19,2)$ \\
\hline
\end{tabular}

Tabla 2 (cont.).

\begin{tabular}{|c|c|c|c|}
\hline & \multicolumn{3}{|c|}{ Prueba estadística } \\
\hline & $\begin{array}{c}\text { Efecto de interacción } \\
\text { (Tiempo*Género) } \\
\text { Wilks Lambda, } \boldsymbol{F}, \boldsymbol{p}\end{array}$ & $\begin{array}{c}\text { Efecto principal } \\
\text { para Tiempo } \\
\text { Wilks Lambda, } F, p\end{array}$ & $\begin{array}{c}\text { Efecto principal } \\
\text { para Género } \\
F, p\end{array}$ \\
\hline \multicolumn{4}{|l|}{ [media $(d t)]$} \\
\hline CPD & $0,970,(3,71) 0,741,0,531$ & $0,298,(3,71) 55,683,<0,0005$ & ,005, ,942 \\
\hline Nivel de $\mathrm{CO}$ en aliento & $0,936,(3,68) 0,882,0,455$ & $0,586,(3,68) 16,037,<0,0005$ & $1,349,, 249$ \\
\hline \multirow[t]{3}{*}{ Puntuaciones en GN-SBQ } & $0,949,(3,69) 1,238,0,303$ & $0,434,(3,69) 30,047,<0,0005$ & $, 00,, 985$ \\
\hline & \multirow{2}{*}{$\begin{array}{l}\text { Entre género } \\
\text { chi cuadrado, } p\end{array}$} & \multicolumn{2}{|c|}{$\begin{array}{c}\text { Intra género } \\
\text { chi cuadrado, } p\end{array}$} \\
\hline & & Hombres & Mujeres \\
\hline \multicolumn{4}{|l|}{ Fumador ${ }^{\mathrm{a}}$ [n (\%)] } \\
\hline Abstinente & Inicio del estudio: ,687, ,407 & & \\
\hline Moderado & S24: ,356, ,949 & $\mathrm{S} 12-24: 2,771,, 428$ & S12-24: $1,590,, 662$ \\
\hline Grave & S36: 4,574, ,206 & S24-36: $2,251,, 522$ & S24-36: ,587, ,899 \\
\hline
\end{tabular}

Nota. CO: monóxido de carbono; CPD: cigarrillos por día; FTND: test de Fagerström de dependencia de nicotina; GN-SBQ: test de Glover-Nilsson; PTN: parches transdérmicos de nicotina; $d t$ : desviación típica; S: semana.

a Estado de tabaquismo autoinformado Abstinente: CPD o, leve: CPD 1-10, moderado: CPD 11-20, grave: CPD > 20

${ }^{\mathrm{b}}$ F se presenta como: (Hipótesis gl, Error $g l$ ) valor F.

\section{Seguridad y tolerabilidad}

Durante el tratamiento activo de 12 semanas, ninguno de los pacientes tuvo intentos de suicidio ni hospitalizaciones (Tabla 3). Hubo un aumento significativo en el perímetro abdominal en los hombres [de 105,98 (DT 13,28) a 108,52 (DT 14,01), $t=-3,436, p=, 002)]$, pero no en las mujeres.

No hubo diferencias de género significativas en los eventos adversos. Los más frecuentes fueron el estreñimiento (14,3 hombres, $26,9 \%$ mujeres), sueños anormales/vívidos (18,4 hombres, 15,4\% mujeres), náuseas/vómitos (10,2 hombres, 26,9\% mujeres) y erupción cutánea/enrojecimiento alrededor de la zona del parche (14,3 hombres, $15,4 \%$ mujeres).

\section{Variables relacionadas con el cese}

En la semana 12, el éxito en el cese del tabaquismo en los hombres se asoció con: menor proporción de antecedentes de suicidio $(p=, 020)$ y menor gravedad psicopatológica $(p=, 020)$ y dependencia psicológica de la nicotina $(p=, 011)$ al inicio del estudio. En las mujeres, se asoció con estar casada o vivir en pareja $(p=, 047)$.

\section{Discusión}

Este estudio es el primero en examinar las diferencias de género en la eficacia, seguridad y tolerabilidad del McSCSP diseñado específicamente para el tratamiento de pacientes con TMG en condiciones clínicas reales. Ha de- 
María José Jaén, María Paz García-Portilla, Fernando Sarramea, Teresa Bobes, Gonzalo Galván, Eva María Díaz-Mesa, Susana Al-Halabi, Edorta Elizagarate, Pilar Alejanda Sáiz, Julio Bobes, Leticia García-Álvarez

Tabla 3. Seguridad en hombres y mujeres.

\begin{tabular}{|c|c|c|c|}
\hline & \multicolumn{3}{|c|}{ Hombres } \\
\hline & $\begin{array}{l}\text { Inicio del estudio } \\
\text { Media (dt) }\end{array}$ & $\begin{array}{l}\text { Semana-12 } \\
\text { Media (dt) }\end{array}$ & Prueba $t$ emparejada, $p$ \\
\hline \multicolumn{4}{|l|}{ PANSS } \\
\hline - PANSS-Positiva & $11,5(3,8)$ & $10,7(3,9)$ & $2,252,, 030$ \\
\hline - PANSS-Negativa & $15,1(5,0)$ & $14,5(5,5)$ & $1,812,, 078$ \\
\hline - PANSS-Psicopatología general & $26,3(5,9)$ & $24,2(6,0)$ & $2,840,, 007$ \\
\hline HDRS & $5,6(4,3)$ & $4,9(5,7)$ & $, 381,, 712$ \\
\hline YMRS & $1,9(2,7)$ & $2,0(4,6)$ & $-, 086,, 933$ \\
\hline CGI-S & $3,6(1,0)$ & $3,5(1,0)$ & $, 206,, 837$ \\
\hline Peso $(k g)$ & $89,2(18,5)$ & $90,7(18,7)$ & $-3.371,0.002$ \\
\hline $\mathrm{IMC}\left(\mathrm{kg} / \mathrm{m}^{2}\right)$ & $30,3(5,8)$ & $30,9(5,8)$ & $-4.126,<0.0005$ \\
\hline Frecuencia cardiaca (lpm) & $81,8(15,6)$ & $82,3(17,8)$ & $0.297,0.768$ \\
\hline \multicolumn{4}{|l|}{ Tensión arterial } \\
\hline Creatinina (mg/dL) & ,9 (,1) & ,9 (,1) & $1,807,, 077$ \\
\hline Urea $(\mathrm{mg} / \mathrm{dL})$ & $29,2(7,9)$ & $29,8(8,0)$ & $-, 813,, 420$ \\
\hline Tasa de filtración glomerular $\mathrm{mL} / \mathrm{min}$ por $1,73 \mathrm{~m}^{2}$ & $100,9(18,7)$ & $101,4(18,9)$ & $-, 329,, 744$ \\
\hline AST $(U / L)$ & $21,6(9,1)$ & $22,4(8,7)$ & $-1,155,, 254$ \\
\hline $\operatorname{ALT}(U / L)$ & $28,5(17,0)$ & $32,6(21,1)$ & $-1,933,, 059$ \\
\hline $\mathrm{GGT}(\mathrm{U} / \mathrm{L})$ & $42,2(30,6)$ & $43,4(30,0)$ & $-, 678,, 501$ \\
\hline Total bilirrubina (mg/dL) &, $5(, 2)$ & ,4 (,2) & $1,033,, 308$ \\
\hline $\operatorname{ALP}(\mathrm{U} / \mathrm{L})$ & $71,3(21,0)$ & $70,3(20,9)$ & $, 990,, 327$ \\
\hline Colesterol (mg/dL) & $207,2(43,9)$ & $198,3(44,5)$ & $2,281,, 027$ \\
\hline HDL-colesterol (mg/dL) & $40,5(10,7)$ & $41,4(11,1)$ & $-1,300,, 200$ \\
\hline LDL-colesterol (mg/dL) & $134,3(41,9)$ & $120,8(40,9)$ & $2,823,, 009$ \\
\hline Triglicéridos (mg/dL) & $202,6(142,5)$ & $224,4(158,8)$ & $-1,357,, 184$ \\
\hline
\end{tabular}

Tabla 3 (cont.).

\begin{tabular}{|c|c|c|c|}
\hline & \multicolumn{3}{|c|}{ Mujeres } \\
\hline & $\begin{array}{l}\text { Inicio del estudio } \\
\text { Media (dt) }\end{array}$ & $\begin{array}{l}\text { Semana-12 } \\
\text { Media (dt) }\end{array}$ & Prueba $t$ emparejada, $p$ \\
\hline \multicolumn{4}{|l|}{ PANSS } \\
\hline - PANSS-Positiva & $11,1(4,0)$ & $8,9(2,2)$ & $2,219,, 044$ \\
\hline - PANSS-Negativa & $14,4(7,1)$ & $15,0(7,6)$ & $-, 402,, 694$ \\
\hline - PANSS-Psicopatología general & $29,5(12,4)$ & $24,3(7,0)$ & $1,549,, 144$ \\
\hline HDRS & $5,0(3,8)$ & $4,8(4,8)$ & $0.210,0.838$ \\
\hline YMRS & $4,1(1,9)$ & $2,7(3,9)$ & $1.288,0.230$ \\
\hline CGI-S & $3,5(1,0)$ & $3,3(1,0)$ & $1.309,0.203$ \\
\hline Peso (kg) & $79,4(14,2)$ & $81,9(14,1)$ & $-3.375,0.003$ \\
\hline $\mathrm{IMC}\left(\mathrm{kg} / \mathrm{m}^{2}\right)$ & $31,5(5,7)$ & $32,6(5,8)$ & $-3.594,0.002$ \\
\hline Frecuencia cardiaca (lpm) & $85,6(16,4)$ & $81,3(15,6)$ & $1.456,0.160$ \\
\hline \multicolumn{4}{|l|}{ Tensión arterial } \\
\hline Creatinina (mg/dL) &, $7(, 1)^{\star}$ &, $7(, 1)^{\star}$ & $-2,119,, 045$ \\
\hline Urea $(\mathrm{mg} / \mathrm{dL})$ & $34,6(9,9)$ & $33,7(10,4)$ & $, 571,, 574$ \\
\hline Tasa de filtración glomerular $\mathrm{mL} / \mathrm{min}$ por $1,73 \mathrm{~m}^{2}$ & $98,2(23,3)$ & $92,4(17,1)$ & $2,191,, 043$ \\
\hline AST $(\mathrm{U} / \mathrm{L})$ & $17,5(5,6)$ & $19,3(8,4)$ & $-1,187,, 248$ \\
\hline $\operatorname{ALT}(\mathrm{U} / \mathrm{L})$ & $19,8(8,2)$ & $22,3(15,4)$ & $-, 738,, 468$ \\
\hline $\mathrm{GGT}(\mathrm{U} / \mathrm{L})$ & $31,0(18,6)$ & $31,4(18,9)$ & $-, 387,, 702$ \\
\hline Total bilirrubina (mg/dL) &, $4(, 2)^{\star \star}$ &, $4(, 2)^{\star \star}$ & $-2,127,, 045$ \\
\hline $\operatorname{ALP}(U / L)$ & $76,2(19,9)$ & $73,8(18,7)$ & $1,470,, 155$ \\
\hline Colesterol (mg/dL) & $206,4(39,2)$ & $209,7(36,9)$ & $-, 944,, 355$ \\
\hline HDL-colesterol (mg/dL) & $51,8(10,8)$ & $52,6(10,5)$ & $-, 796,, 434$ \\
\hline LDL-colesterol (mg/dL) & $132,4(29,7)$ & $129,4(31,3)$ & $, 522,, 610$ \\
\hline Triglicéridos (mg/dL) & $134,8(109,8)$ & $148,3(104,0)$ & $-1,522,, 149$ \\
\hline
\end{tabular}

Nota. ALP: fosfatasa alcalina; ALT: alanina aminotransferasa; AST: aspartato aminotransferasa; IMC: índice de masa corporal; CGI-S: escala de impresión clínica global - severidad; GGT: gamma glutamil transferasa; HDL: lipoproteínas de alta densidad; HDRS: escala de depresión de Hamilton; LDL: lipoproteínas de baja densidad; PANSS: Escala de Síntomas Positivos y Negativos; dt: desviación típica; YMRS: escala de manía de Young. *Inicio del estudio o,7096 (o,11767); Semana-12 o,7308 $(0,10480) ;{ }^{\star \star} 0,3565(0,17629) ; 0,3700(0,18918)$. 
mostrado efectividad en hombres y mujeres. No hubo diferencias entre los grupos en las tasas de cese del tabaquismo en ningún momento, ni controlando por diagnóstico o tratamiento.

No hubo diferencias de género significativas en una serie de variables relacionadas con el tabaquismo al inicio del estudio. Como en estudios anteriores, encontramos menos diferencias de género en los fumadores con TMG en comparación con los de la población general (Filia et al., 2014). Las personas con TMG (hombres y mujeres) tienen tasas más altas de tabaquismo (Kumari y Postma, 2005) y dependencia de nicotina que los fumadores de la población general, fuman más cigarrillos por día y tienen puntuaciones más elevadas en la prueba FTND (Gurpegui et al., 2005), posiblemente, contribuyendo a una mayor homogeneidad entre los fumadores con trastornos graves (Filia et al., 2014).

Como se ha observado en los pocos estudios previos llevados a cabo con pacientes con enfermedades mentales (Filia et al., 2014), no hay diferencias de género en las tasas de cesación, a diferencia de lo observado en la población general donde las mujeres tienen más dificultad para dejar de fumar y muestran resultados más pobres en el tratamiento para dejar de fumar (McKee, O’Malley, Salovey, Krishnan-Sarin y Mazure, 2005; Perkins y Scott, 2008; Reid et al., 2009; Smith, Bessette, Weinberger, Sheffer y McKee, 2016). Sin embargo, a pesar de esta falta de diferencias significativas, la tasa de cese tabáquica a las 12 semanas es aparentemente mayor en las mujeres. A las 24 y 36 semanas, la tasa de cese del tabaquismo es aparentemente la misma. Estos resultados son interesantes porque parece ser que los hombres que logran la abstinencia a corto plazo la mantienen con el tiempo, mientras que un alto porcentaje de mujeres reanudan el hábito después de la abstinencia $(15,4 \%$ vs $4,1 \%)$.

No hubo empeoramiento psicopatológico, intentos de suicidio ni hospitalizaciones. Además, los hombres mejoraron más que las mujeres, disminuyendo las puntuaciones en la PANSS positiva, psicopatología general y puntuación total, mientras que las mujeres mejoraron solo en sintomatología positiva. Según estudios previos (Ostacher et al., 2006), existe una asociación entre fumar y una mayor gravedad del trastorno mental, por lo que tal vez la cesación tabáquica de esos pacientes pudo contribuir a esta mejoría. Otros estudios encuentran que puntuaciones totales más altas en la PANSS son menos frecuentes en fumadores con dependencia leve (Aguilar, Gurpegui, Diaz y De Leon, 2005).

Dejar de fumar con frecuencia se asocia con un aumento de peso (Aubin, Farley, Lycett, Lahmek y Aveyard, 2012). En consonancia, se ha observado que tanto los hombres como las mujeres experimentaron aumentos significativos de peso (aproximadamente $4 \mathrm{~kg}$ ) y del IMC, además, en el caso de los hombres también aumentó el perímetro abdominal. Como en estudios previos (Filia, Baker, Gurvich, Richmond y Kulkarni, 2014), no hubo diferencias de género con respecto al aumento de peso.

En este estudio, las variables relacionadas con el cese exitoso del tabaquismo en la semana 12 en los hombres fueron la falta de antecedentes de suicidio y una menor dependencia psicológica de la nicotina, así como la gravedad psicopatológica (CGI-S) al inicio del estudio. En las mujeres, la variable relacionada con éxito para dejar de fumar fue el estado civil, específicamente estar casada o vivir en pareja. En los hombres y mujeres con enfermedad mental, el nivel basal de CO espirado y un mayor número de visitas al programa han demostrado ser predictivos de la cesación tabáquica, mientras que en los hombres, los antecedentes de uso de alcohol, heroína, otros opioides y marihuana fueron predictores de la falta de éxito para dejar de fumar (Okoli et al., 2011). El conocimiento sobre los factores relacionados con el abandono exitoso del tabaquismo puede ser importante en el diseño de futuros programas para dejar de fumar en personas con TMG.

Nuestros resultados deben interpretarse teniendo en cuenta una serie de limitaciones metodológicas. Primero, el tamaño muestral relativamente pequeño en cada grupo puede estar relacionado con la ausencia de mayor número de diferencias de género. Es por este motivo que no se dividió cada grupo de género por diagnóstico, a pesar de las diferencias significativas. En segundo lugar, este estudio se realizó con una muestra de fumadores con psicosis y trastorno bipolar, por lo que no se trata de un grupo homogéneo.

En conclusión, hemos demostrado que este programa es efectivo y seguro para ayudar tanto a los hombres como a las mujeres con TMG estabilizados a dejar de fumar. Se requiere investigación futura con muestras de mayor tamaño para determinar con más claridad la existencia de diferencias, además de la fiabilidad y robustez.

\section{Reconocimientos}

Este estudio se realizó sin apoyo financiero de ninguna compañía farmacéutica. Obtuvo apoyo parcial del Ministerio de Ciencia e Innovación de España, el Instituto de Salud Carlos III (ayuda PI10/01758 concedida a Dr. Julio Bobes), el Gobierno del Principado de Asturias PCTI-20182022 IDI/2018/235, el CIBERSAM y los Fondos Europeos de Desarrollo Regional (FEDER). Los Servicios de Salud del Principado de Asturias, España, donaron parte de la medicación utilizada en este estudio.

Los autores desean expresar su gratitud a la Fundación para la Investigación e Innovación Biosanitaria del Principado de Asturias (Finba) por su apoyo financiero. 


\section{Referencias}

Aguilar, M. C., Gurpegui, M., Diaz, F. J. y De Leon, J. (2005). Nicotine dependence and symptoms in schizophrenia. British Journal of Psychiatry, 186, 215-221. doi:10.1192/ bjp.186.3.215.

Aubin, H.-J., Farley, A., Lycett, D., Lahmek, P. y Aveyard, P. (2012). Weight gain in smokers after quitting cigarettes: Meta-analysis. BMJ, 345, 4439-4439. doi:10.1136/bmj. e4439.

Bjornson, W., Rand, C., Connett, J. E., Lindgren, P. Nides, M., Pope, F.,... O'Hara, P. (1995). Gender differences in smoking cessation after 3 years in the Lung Health Study. American Journal of Public Health, 85, 223-230. doi:10.2105/ajph.85.2.223.

COMMIT. (1995). Community Intervention Trial for Smoking Cessation (COMMIT): I. cohort results from a fouryear community intervention. American Journal of Public Health, 85, 183-192. doi:10.2105/ajph.85.2.183.

Davis, L. J. J., Hurt, R. D., Offord, K. P., Lauger, G. G., Morse, R. M. y Bruce, B. K. (1994). Self-administered Nicotine-Dependence Scale (SANDS): Item selection, reliability estimation, and initial validation. Journal of Clinical Psychology, 50, 918-930. doi:10.1002/1097-4679(199411) 50:6<918::aid-jclp2270500617>3.0.co;2-6.

De Hert, M., Correll, C. U., Bobes, J., Cetkovich-Bakmas, M., Cohen, D. A. N., Asai, I.,... Leucht, S. (2011). Physical illness in patients with severe mental disorders. I. Prevalence, impact of medications and disparities in health care. World Psychiatry, 10, 52-77. doi:10.1002/j.2051-5545.2011.tb00014.x.

Filia, S. L., Baker, A. L., Gurvich, C. T., Richmond, R. y Kulkarni, J. (2014). The perceived risks and benefits of quitting in smokers diagnosed with severe mental illness participating in a smoking cessation intervention: Gender differences and comparison to smokers without mental illness. Drug and Alcohol Review, 33, 78-85. doi:10.1111/dar.12091.

Filia, S. L., Baker, A. L., Gurvich, C. T., Richmond, R., Lewin, T. J. y Kulkarni, J. (2014). Gender differences in characteristics and outcomes of smokers diagnosed with psychosis participating in a smoking cessation intervention. Psychiatry Research, 215, 586-593. doi:10.1016/j.psychres.2014.01.002.

Garcia-Portilla, M. P., Garcia-Alvarez, L., Saiz, P. A., Diaz-Mesa, E., Galvan, G., Sarramea, F.,... Bobes, J. (2014). Effectiveness of a multi-component Smoking Cessation Support Programme (McSCSP) for patients with severe mental disorders: Study design. International Journal of Environmental Research and Public Health, 11, 373-389. doi:10.3390/ijerph110100373.

Garcia-Portilla, M. P., Garcia-Alvarez, L., Sarramea, F., Galvan, G., Diaz-Mesa, E., Bobes-Bascaran, T.,... Bobes, J. (2016). It is feasible and effective to help patients with severe mental disorders to quit smoking: An ecological pragmatic clinical trial with transdermal nicotine patches and varenicline. Schizophrenia Research, 176, 272280. doi:10.1016/j.schres.2016.05.011.

Gurpegui, M., Martínez-Ortega, J. M., Aguilar, M. C., Diaz, F. J., Quintana, H. M. y De Leon, J. (2005). Smoking initiation and schizophrenia: A replication study in a Spanish sample. Schizophrenia Research, 76, 113-118. doi:10.1016/j.schres.2004.12.007.

Hahn, L., Rigby, A. y Galletly, C. (2014). Determinants of high rates of smoking among people with psychosis living in a socially disadvantaged region in South Australia. Australian and New Zealand Journal of Psychiatry, 48, 70-79. doi:10.1177/0004867413491158.

Jiménez-Treviño, L., Velasco, Á., Rodriguez-Revuelta, J., Abad, I., De la Fuente-Tomás, L., González-Blanco, L. y Sáiz, P. A. (2019). Factors associated with tobacco consumption in patients with depression. Adicciones, 31, 298-308. doi:10.20882/adicciones.1191.

Kim, S. S., Chung, S., Park, J. Il, Jung, A. J., Kalman, D. y Ziedonis, D. M. (2013). Smoking among individuals with schizophrenia in korea: Gender differences. $A r$ chives of Psychiatric Nursing, 27, 241-245. doi:10.1016/j. apnu.2013.06.002.

Kumari, V. y Postma, P. (2005). Nicotine use in schizophrenia: The self medication hypotheses. Neuroscence y Biobehavioral Reviews, 29, 1021-1034. doi:10.1016/j.neubiorev.2005.02.006.

McKee, S. A., O’Malley, S. S., Salovey, P., Krishnan-Sarin, S. y Mazure, C. M. (2005). Perceived risks and benefits of smoking cessation: Gender-specific predictors of motivation and treatment outcome. Addictive Behaviors, 30, 423-435. doi:10.1016/j.addbeh.2004.05.027.

Okoli, C. T. C., Khara, M., Torchalla, I., Ensom, M. H. H., Oliffe, J. L., Bottorff, J. L. y Stanley, P. J. (2011). Sex differences in smoking cessation outcomes of a tailored program for individuals with substance use disorders and mental illness. Addictive Behaviors, 36, 523-526. doi:10.1016/j.addbeh.2010.12.029.

Ostacher, M. J., Nierenberg, A. A., Perlis, R. H., Eidelman, P., Borrelli, D. J., Tran, T. B.,... Sachs, G. S. (2006). The relationship between smoking and suicidal behavior, comorbidity, and course of illness in bipolar disorder. The Journal of Clinical Psychiatry, 67, 1907-1911. doi:10.4088/ jcp.v67n1210.

Perkins, K. A. (2001). Smoking cessation in women. Special considerations. CNS Drugs, 15, 391-411. doi:10.2165/00023210-200115050-00005.

Perkins, K. A. y Scott, J. (2008). Sex differences in long-term smoking cessation rates due to nicotine patch. Nicotine and Tobacco Research, 10, 1245-1251. doi:10.1080/14622200802097506.

Reid, R. D., Pipe, A. L., Riley, D. L. y Sorensen, M. (2009). Sex differences in attitudes and experiences concerning smoking and cessation: Results from an international 
survey. Patient Education and Counseling, 76, 99-105. doi:10.1016/j.pec.2008.11.001.

Rodriguez Muñoz, P. M., Carmona Torres, J. M., Hidalgo Lopezosa, P., Cobo Cuenca, A. I. y Rodríguez Borrego, M. A. (2019). Evolution of alcohol and tobacco consumption in young people in Spain, after the law 42/2010 against smoking: 2011-2014. Adicciones, 31, 274283. doi:10.20882/adicciones.1035.

Sarramea Crespo, F., Jaén-Moreno, M. J., Gutiérrez-Rojas, L., Balanzá-Martínez, V., García-Alvarez, L., Saiz Martínez, P. A. y Bobes, J. (2019a). "Readiness to change" predicts efficacy of reduction among smokers with severe mental illness. European Addiction Research, 25, 256262. doi:10.1159/000500450.

Sarramea, F., Jaen-Moreno, M. J., Balanza-Martinez, V., Osuna, M. I., Alcala, J. A., Montiel, F. J.,... Gutierrez-Rojas, L. (2019b). Setting the stage to quit smoking in Bipolar Disorder patients: Brief advice in clinical practice. Adicciones, 31,136-146. doi:10.20882/adicciones.1006.

Scharf, D. y Shiftman, S. (2004). Are there gender differences in smoking cessation, with and without bupropion? Pooled- and meta-analyses of clinical trials of Bupropion SR. Addiction, 99, 1462-1469. doi:10.1111/j.13600443.2004.00845.x.

Smith, P. H., Bessette, A. J., Weinberger, A. H., Sheffer, C. E. y McKee, S. A. (2016). Sex/gender differences in smoking cessation: A review. Preventive Medicine, 92, 135-140. doi:10.1016/j.ypmed.2016.07.013.

Walker, N. J., Van Woerden, H. C., Kiparoglou, V., Yang, Y., Robinson, H. y Croghan, E. (2016). Gender difference and effect of pharmacotherapy: Findings from a smoking cessation service. BMC Public Health, 16, 1-8. doi:10.1186/s12889-016-3672-y.

Wetter, D. W., Kenford, S. L., Smith, S. S., Fiore, M. C., Jorenby, D. E. y Baker, T. B. (1999). Gender differences in smoking cessation. Journal of Consulting and Clinical Psychology, 67, 555-562. doi:10.1037//0022-006x.67.4.555. 